\title{
Recurrent sets and shadowing for finitely generated semigroup actions on metric spaces
}

\author{
Zahra Shabani ${ }^{1}$ (D), Ali Barzanouni ${ }^{2}$ (D), Xinxing $\mathrm{Wu}^{* 3}$ (D) \\ ${ }^{1}$ Department of Mathematics, Faculty of Mathematics, Statistics and Computer Science, University of \\ Sistan and Baluchestan, Zahedan, Iran \\ ${ }^{2}$ Department of Mathematics, Hakim Sabzevari University, Sabzevar, Iran \\ ${ }^{3}$ School of Mathematics and Statistics, Guizhou University of Finance and Economics, Guiyang 550025, \\ China
}

\begin{abstract}
We introduce various new type of recurrent sets for finitely generated semigroups on noncompact metric spaces that are conjugacy invariant, and obtain some basic properties of chain recurrent sets for semigroups via these new definitions. Moreover, we define the notion of weak shadowing property for finitely generated group actions on compact metric spaces, which is weaker than that of shadowing property, and prove the equivalence of the shadowing and weak shadowing properties for the finitely generated group actions on a generalized homogeneous space without isolated points.
\end{abstract}

Mathematics Subject Classification (2020). 54H20, 37B20, 37C50

Keywords. group actions, recurrent sets, shadowing property, weak shadowing property

\section{Introduction}

The notion of shadowing was introduced in the 1970s independently by Anosov [3] and Bowen [6]. A dynamical system has the shadowing property if every sufficiently precise trajectory is closed to some exact trajectory. The shadowing property has been developed intensively in recent years, and many authors obtained results about chaos and stability by studying the various type of shadowing (see $[1,11,17,19,20,22,24,25,27-29]$ ). Wu et al. [25] introduced the notion of $\mathscr{M}_{\alpha}$-shadowing and proved that a dynamical system has the average shadowing property if and only if it has the $\mathscr{M}_{\alpha}$-shadowing property for any $\alpha \in[0,1)$. Oprocha and $\mathrm{Wu}$ [17] proved that a dynamical system with the average shadowing of periodic pseudo-orbits property is distributionally chaotic if and only if it has a distal pair. Lewowicz [14] introduced the concept of persistence for dynamical systems which is weaker than that of topological stability. Artigue [4] showed that if a bi-Lipschitz homeomorphism on a compact manifold is persistent or has the weak shadowing property, then it is Lipschitz structurally stable. Zhang and $\mathrm{Wu}[29]$ obtained that the $C^{1}$-stably $\mathscr{M}_{0}$-shadowing property on a non-trivial transitive set implies the diffeomorphism has a dominated splitting, extending main results in [20].

\footnotetext{
*Corresponding Author.

Email addresses: zshabani@math.usb.ac.ir (Z. Shabani), A.barzanouni@hsu.ac.ir (A. Baranouni), wuxinxing5201314@163.com (X. Wu)

Received: 22.08.2020; Accepted: 01.02.2021
} 
Throughout this paper, let $\mathbb{N}=\{1,2,3, \ldots\}$ and $\mathbb{Z}^{+}=\{0,1,2, \ldots\}$. Let $(X, d)$ be a metric space, $i d$ be the identity map on $X$, and each $g_{i}: X \rightarrow X(i=1, \ldots, m)$ is a continuous self-maps on $X$. The finitely generated semigroup $(G, \circ)$ generated by $G_{1}=\left\{i d, g_{1}, \ldots, g_{m}\right\}$ is defined by $G=\bigcup_{n \in \mathbb{Z}^{+}} G_{n}$, where $G_{0}=i d$ and

$$
G_{n}=\left\{g_{i_{n}} \circ \cdots \circ g_{i_{1}}: g_{i_{j}} \in G_{1}\right\}, n \geq 1 \text {. }
$$

Indeed, each element of $G_{n}$ is a composition of at most $n$ elements of $G_{1}$. We mention that the finitely generated semigroup action is also called iterated function system.

Recently, Osipov and Thikhomirov [18] introduced the notion of shadowing property for finitely generated group action. Chung and Lee [9] studied the topological stability and pseudo-orbit tracing property for group actions. Ahn et al. [2] introduced the notion of persistent actions for finitely generated group action on compact metric spaces and studied its relation to the topological stability. Bahabadi [5] discussed the shadowing and average shadowing properties for iterated function systems. Wu et al. [26] further studied some chain properties and average shadowing for iterated function systems and proved that an iterated function system with (asymptotic) average shadowing is chain mixing. For more recent results on finitely generated group or semigroup actions on compact metric spaces, we refer the reader to $[7,10,12,15,23,30]$ and references therein.

However, the definitions of chain recurrent sets and shadowing property for continuous map $f$ and also for the finitely generated semigroup actions on a compact metric space depend on the metrics on non-compact metric spaces. In other words, a point in chain recurrent set of $f$ (or semigroup $G$ ) with respect to one metric, may not be in chain recurrent set of $f$ (or semigroup $G$ ) with respect to another metric inducing the same topology (see [13, Example 2.2] and Example 3.1). Lee et al. [13] introduced the notions of $\varepsilon$-chain and shadowing property for homeomorphisms on non-compact metric spaces, which are dynamical properties and equivalent to the classical definitions in case of compact metric spaces, and extended Walters's stability theorem to homeomorphisms on locally compact metric spaces.

In the present paper, we extend the notion of $\varepsilon$-chain defined in [13] to the case of finitely generated semigroup actions on non-compact metric spaces and show that the new notion of $\varepsilon$-chain on a non-compact metric space $X$ is independent of metrics on $X$. Afterwards, we extend the notion of weak shadowing property from homeomorphisms to finitely generated semigroup actions on compact metric spaces, and we show that while the shadowing property implies the weak shadowing property, there exists a semigroup on a compact metric space $X$ that has the weak shadowing property but does not have the shadowing property. Furthermore, we obtain the equivalence of shadowing and weak shadowing properties on generalized homogeneous spaces without the isolated points.

This paper is organized as follows. In Section 2, we extend the notion of weakly periodic points and weakly non-wandering points defined in [8] to the case of finitely generated semigroup actions on compact metric spaces and obtain some basic properties on them, and chain recurrent sets for finitely generated semigroup. In Section 3, we first introduce various new type of recurrent sets for finitely generated semigroups on non-compact metric spaces that are conjugacy invariant, and then study some properties of chain recurrent sets for finitely generated semigroup via these new definitions. In Section 4, we introduce the notion of weak shadowing property for finitely generated semigroups on compact metric spaces and build an example showing that the shadowing property is strictly stronger than the weak shadowing property on compact metric spaces. Moreover, we prove that they are equivalent on generalized homogeneous spaces without isolated points.

\section{Various type of recurrent sets on compact metric spaces}

Let $G$ be a semigroup generated by the finite family $G_{1}=\left\{i d, g_{1}, \ldots, g_{m}\right\}$. Symbolic dynamic is a way to display the elements of the semigroup $G$. Let $\Sigma_{m}$ be the space of 
two sided infinite sequences of $m$ symbols $\{1, \ldots, m\}$, that is, $\Sigma_{m}=\{1, \ldots, m\}^{\mathbb{Z}}$. For any sequence $\omega=\cdots \omega_{-2} \omega_{-1} \omega_{0} \omega_{1} \omega_{2} \cdots \in \Sigma_{m}$, take $G_{\omega}^{0}:=i d$ and, for any $n \in \mathbb{N}$,

$$
G_{\omega}^{n}(x):=g_{\omega_{n-1}} \circ \cdots \circ g_{\omega_{0}}(x) .
$$

In particular, if the elements of $G_{1}$ are invertible, we take $G_{\omega}^{0}:=i d$ and, for any $n>0$,

$$
G_{\omega}^{n}(x):=g_{\omega_{n-1}} \circ \cdots \circ g_{\omega_{0}}(x), G_{\omega}^{-n}(x):=g_{\omega_{-n}}^{-1} \circ \cdots \circ g_{\omega_{-1}}^{-1}(x) .
$$

Let $\mathcal{A}_{m}$ be the set of all finite words of symbols $\{1, \ldots, m\}$, that is,

$$
\mathcal{A}_{m}=\bigcup_{n \in \mathbb{N}}\{1, \ldots, m\}^{n} .
$$

We use the notation $|w|$ for the length of $w \in \mathcal{A}_{m}$. For any $w=w_{1} \cdots w_{n} \in \mathcal{A}_{m}$, we denote $G_{w}^{0}=i d, G_{w}=g_{w_{n}} \circ \cdots \circ g_{w_{1}}$, and $G_{w}^{i}:=g_{w_{i}} \circ \cdots \circ g_{w_{1}}$ for $1 \leq i \leq n$. Clearly, $G_{w}=G_{w}^{|w|}$.

Let $(X, d)$ be a compact metric space and $G$ be a semigroup generated by the finite family $\left\{i d, g_{1}, \ldots, g_{m}\right\}$ of continuous self-maps on $X$. For $w=w_{0} \cdots w_{n-1} \in \mathcal{A}_{m}$ and $\varepsilon>0$, an $(\varepsilon, w)$-chain of semigroup $G$ from $x$ to $y$ is a finite sequence $\left\{x_{0}=x, x_{1}, \ldots, x_{n}=y\right\}$ satisfying

$$
d\left(g_{w_{i}}\left(x_{i}\right), x_{i+1}\right)<\varepsilon \text { for } 0 \leq i \leq n-1 .
$$

An $\varepsilon$-chain of semigroup $G$ from $x$ to $y$ is an $(\varepsilon, w)$-chain from $x$ to $y$ for some $w \in \mathcal{A}_{m}$.

We say that a subset $\Lambda \subset X$ is invariant for the semigroup $G$ if $g_{i}(\Lambda) \subset \Lambda$ for $1 \leq i \leq m$.

Let $(X, d)$ be a compact metric space and $C(X)$ be the collection of all continuous self-maps on $X$ with the following $C^{0}$-metric $d_{0}$ :

$$
d_{0}(f, g)=\max _{x \in X} d(f(x), g(x)) .
$$

Let $C_{m}(X)$ be the collection of all semigroups such as $G$ on a metric space $X$ which has a finite set of generators $\left\{i d, g_{1}, \ldots, g_{m}\right\}$, where $g_{i} \in C(X), i=1, \ldots, m$. Given two semigroups $F, G \in C_{m}(X)$ generated by the finite families $F_{1}=\left\{i d, f_{1}, \ldots, f_{m}\right\}$ and $G_{1}=\left\{i d, g_{1}, \ldots, g_{m}\right\}$, respectively, define the $C^{0}$-metric $D_{0}$ on $C_{m}(X)$ by

$$
D_{0}(F, G)=\max _{1 \leq i \leq m} d_{0}\left(f_{i}, g_{i}\right) \text {. }
$$

Also, we say that $F$ is $\varepsilon$-close to $G$ if $D_{0}(F, G)<\varepsilon$.

Definition 2.1. Let $(X, d)$ be a compact metric space and $G \in C_{m}(X)$ be a semigroup generated by $G_{1}=\left\{i d, g_{1}, \ldots, g_{m}\right\}$. A point $x \in X$ is

(i) a periodic point of the semigroup $G$ if there exists some $w \in \mathcal{A}_{m}$ such that $G_{w}^{|w|}(x)=$ $x$.

(ii) a weakly periodic point of the semigroup $G$ if, for any $\varepsilon>0$, there exists a semigroup $F \in C_{m}(X)$ such that $D_{0}(F, G)<\varepsilon$ and $x$ is a periodic point of $F$.

(iii) a chain recurrent point of semigroup $G$ if, for any $\varepsilon>0$, there exists an $\varepsilon$-chain of the semigroup $G$ from $x$ to itself.

(iv) a non-wandering point of the semigroup $G$ if, for every open neighborhood $U$ of $x$, there exist $n \in \mathbb{N}$ and $\omega \in \Sigma_{m}$ such that $G_{\omega}^{n}(U) \cap U \neq \varnothing$.

(v) a weakly non-wandering point of the semigroup $G$ if, for any $\varepsilon>0$, there exists a semigroup $F \in C_{m}(X)$ such that $D_{0}(F, G)<\varepsilon$ and $x$ is a non-wandering point of $F$.

The sets of periodic points, weakly periodic points, chain recurrent points, non-wandering points, and weakly non-wandering points of the semigroup $G$ are denoted by $\operatorname{Per}(G)$, $\operatorname{Per}_{\mathrm{w}}(G), C R(G), \Omega(G)$, and $\Omega_{\mathrm{w}}(G)$, respectively.

Remark 2.2. (1) From Definition 2.1, it is clear that $\operatorname{Per}(G) \subseteq \operatorname{Per}_{\mathrm{w}}(G), \Omega(G) \subseteq$ $\Omega_{\mathrm{w}}(G)$, and $\operatorname{Per}_{\mathrm{w}}(G) \subseteq \Omega_{\mathrm{w}}(G), \operatorname{Per}(G) \subseteq \Omega(G)$.

(2) Note that every compact dynamical system contains a minimal point. This implies that for every finitely generated semigroup $G$ on a compact metric space, $\Omega(G) \neq \varnothing$. 
Let $(X, d)$ be a compact metric space and $G \in C_{m}(X)$ be a semigroup generated by $G_{1}=\left\{i d, g_{1}, \ldots, g_{m}\right\}$. Fix $x \in \Omega_{\mathrm{w}}(G)$. Then, for any $\varepsilon>0$, there exists a semigroup $F \in C_{m}(X)$ such that $D_{0}(F, G)<\frac{\varepsilon}{2}$ and $x \in \Omega(F)$. Without loss of generality, assume that $F$ is generated by $\left\{i d, f_{1}, \ldots, f_{m}\right\}$. The uniform continuity of $g_{1}, \ldots, g_{m}$ implies that there exists $0<\delta<\frac{\varepsilon}{2}$ such that for any $x_{1}, x_{2} \in X$ with $d\left(x_{1}, x_{2}\right)<\delta$, $\max _{1 \leq i \leq m} d\left(g_{i}\left(x_{1}\right), g_{i}\left(x_{2}\right)\right)<\frac{\varepsilon}{2}$. Applying $x \in \Omega(F)$ yields that there exist $\omega \in \Sigma_{m}$ and $n \in \mathbb{N}$ such that $F_{\omega}^{n}(B(x, \delta)) \cap B(x, \delta) \neq \varnothing$, where $B(x, \delta)=\{z \in X: d(x, z)<\delta\}$. Take $y \in B(x, \delta)$ with $F_{\omega}^{n}(y) \in B(x, \delta)$. We have

(1) $d\left(G_{\omega}^{1}(x), F_{\omega}^{1}(y)\right)=d\left(g_{\omega_{0}}(x), f_{\omega_{0}}(y)\right) \leq d\left(g_{\omega_{0}}(x), g_{\omega_{0}}(y)\right)+d\left(g_{\omega_{0}}(y), f_{\omega_{0}}(y)\right)<\frac{\varepsilon}{2}+$ $D_{0}(F, G)<\varepsilon$

(2) for $1 \leq i<n-1, d\left(g_{\omega_{i}}\left(F_{\omega}^{i}(y)\right), F_{\omega}^{i+1}(y)\right)=d\left(g_{\omega_{i}}\left(F_{\omega}^{i}(y)\right), f_{\omega_{i}}\left(F_{\omega}^{i}(y)\right)\right) \leq D_{0}(F, G)<\varepsilon$;

(3) $d\left(g_{\omega_{n-1}}\left(F_{\omega}^{n-1}(y)\right), x\right) \leq d\left(g_{\omega_{n-1}}\left(F_{\omega}^{n-1}(y)\right), f_{\omega_{n-1}}\left(F_{\omega}^{n-1}(y)\right)\right)+d\left(f_{\omega_{n-1}}\left(F_{\omega}^{n-1}(y)\right), x\right)=$ $d\left(g_{\omega_{n-1}}\left(F_{\omega}^{n-1}(y)\right), f_{\omega_{n-1}}\left(F_{\omega}^{n-1}(y)\right)\right)+d\left(F_{\omega}^{n}(y), x\right)<D_{0}(F, G)+\delta<\varepsilon$.

This implies that the sequence $\left\{x, F_{\omega}^{1}(y), F_{\omega}^{2}(y), \ldots, F_{\omega}^{n-1}(y), x\right\}$ is an $\varepsilon$-chain of $G$ from $x$ to itself. By this observation, the following result is obtained.

Proposition 2.3. Let $G$ be a finitely generated semigroup on a compact metric space $X$. Then $\Omega_{\mathrm{w}}(G) \subseteq C R(G)$.

Remark 2.4. Choi et al. [8, Example 8] showed that there exists a dynamical system $(X, f)$ such that $\Omega_{\mathrm{w}}(f) \varsubsetneqq C R(f)$.

From Remark 2.2 and Proposition 2.3, it is clear that

$$
\operatorname{Per}(G) \subseteq \Omega(G) \subseteq \Omega_{\mathrm{w}}(G) \subseteq C R(G),
$$

and

$$
\operatorname{Per}_{\mathrm{w}}(G) \subseteq \Omega_{\mathrm{w}}(G) \subseteq C R(G) .
$$

For $\varepsilon>0$ and $x \in X$, let

$$
C_{\varepsilon}(x, G)=\{y \in X \text { : there exists an } \varepsilon \text {-chain from } x \text { to } y\},
$$

and $C(x, G)=\bigcap_{\varepsilon>0} C_{\varepsilon}(x, G)$. Clearly, $x \in C R(G)$ if and only if $x \in C(x, G)$. We say that the finitely generated semigroup $G$ on $X$ is

(1) chain transitive on a non-empty subset $\Lambda$ of $X$ if, for any $x \in \Lambda, C(x, G)=\Lambda$;

(2) chain transitive if it is chain transitive on the whole space $X$.

Applying Remark 2.2, the proof of the following proposition is straightforward and is similar to the classical case for the continuous map $f: X \rightarrow X$, so we omit it.

Proposition 2.5. Let $G$ be a finitely generated semigroup on a compact metric space $X$ and $x \in X$. The following statements hold:

(1) The set $\Omega(G)$ is a nonempty closed subset of $X$.

(2) The set $C R(G)$ is a nonempty closed subset of $X$.

(3) The set $C(x, G)$ is closed and invariant for the semigroup $G$.

Example 2.6. Consider the space $X=\{a, b, c\}$ with discrete metric $d$, where $a, b, c$ are different points. Let $g_{i}: X \rightarrow X$ is defined by $g_{i}(b)=c$ and $g_{i}(c)=c$ for $i=1,2$ and $g_{1}(a)=a$ and $g_{2}(a)=b$. It can be verified that $a \in \operatorname{Per}(G)$ but $g_{2}(a)=b \notin C R(G)$. This, together with (2.1), implies that $\operatorname{Per}(G), \Omega(G), \Omega_{\mathrm{w}}(G)$, and $C R(G)$ may not be invariant.

Next, we show that, for a finitely generated semigroup $G$, the chain transitivity on the non-wandering set $\Omega(G)$ implies the chain transitivity on the whole space.

Proposition 2.7. Let $G$ be a semigroup generated by the finite family $\left\{i d, g_{1}, \ldots, g_{m}\right\}$ of homeomorphisms on a compact metric space $(X, d)$ and $x \in X$. If $\Omega(G) \subseteq C(x, G)$, then $C(x, G)=X$, and therefore $x \in C R(G)$. 
Proof. Fix any $y \in X$. For any fixed $\varepsilon>0$, let $K=\left\{z \in X: \operatorname{dist}(z, \Omega(G)) \geq \frac{\varepsilon}{4}\right\}$, where $\operatorname{dist}(z, \Omega(G))=\inf \{d(z, x): x \in \Omega(G)\}$. For any $\hat{y} \in X \backslash K$, i.e., $\operatorname{dist}(\hat{y}, \Omega(G))<\frac{\varepsilon}{4}$, since $\Omega(G)$ is a nonempty closed set, there exists some $z \in \Omega(G) \subseteq C(x, G)$ such that $d(\hat{y}, z)=d(\hat{y}, \Omega(G))<\frac{\varepsilon}{4}$. From $z \in C(x, G)$, it follows that there exists an $\frac{\varepsilon}{4}$-chain $\left\{x_{0}=x, x_{1}, \ldots, x_{n-1}, x_{n}=z\right\}$ from $x$ to $z$, implying that $\left\{x_{0}=x, x_{1}, \ldots, x_{n-1}, \hat{y}\right\}$ is an $\varepsilon$-chain from $x$ to $\hat{y}$, and thus $X \backslash K \subset C_{\varepsilon}(x, G)$.

For any $z \in K \subset X \backslash \Omega(G)$, it follows from Definition 2.1 that there exists an open neighborhood $U(z)$ of $z$ such that $G_{w}^{n}(U(z)) \cap U(z)=\varnothing$ for any $n \in \mathbb{N}$ and any $w \in \Sigma_{m}$. By the compactness of $K$, there exists a finite set $\left\{z_{1}, z_{2}, \ldots, z_{m_{0}}\right\} \subset K$ such that $K \subseteq$ $\bigcup_{i=1}^{m_{0}} U\left(z_{i}\right)$.

To prove $y \in C_{\varepsilon}(x, G)$, consider the following two cases:

Case 1. If $y \in X \backslash K$, it is clear that $y \in C_{\varepsilon}(x, G)$ as $X \backslash K \subset C_{\varepsilon}(x, G)$;

Case 2. If $y \in K$, we claim that for any $w \in \Sigma_{m}$, there exists $0 \leq j \leq m_{0}$ such that $G_{w}^{-j}(y) \notin K$. Suppose on the contrary that there exists some $w \in \Sigma^{m}$ such that, for any $0 \leq j \leq m_{0}, G_{w}^{-j}(y) \in K$. This implies that $\left\{G_{w}^{-j}(y): 0 \leq j \leq m_{0}\right\} \subset K$. Noting that $K \subseteq \bigcup_{i=1}^{m_{0}} U\left(z_{i}\right)$, applying the pigeon-hole principle yields that there exist two integers $j_{1}, j_{2} \in\left\{0,1, \ldots, m_{0}\right\}$ with $j_{1}<j_{2}$ and $t \in\left\{1, \ldots, m_{0}\right\}$ such that $G_{w}^{-j_{1}}(y), G_{w}^{-j_{2}}(y) \in U\left(z_{t}\right)$. This, together with

$$
\begin{aligned}
& G_{w}^{-j_{2}}(y)=g_{w_{-j_{2}}}^{-1} \circ \cdots \circ g_{w_{-j_{1}}}^{-1} \circ \cdots \circ g_{w_{-1}}^{-1}(y) \\
& =g_{w_{-j_{2}}}^{-1} \circ \cdots \circ g_{w_{-\left(j_{1}+1\right)}}^{-1}\left(G_{w}^{-j_{1}}(y)\right) \in U\left(z_{t}\right) \text {, }
\end{aligned}
$$

implies that $g_{w_{-j_{2}}}^{-1} \circ \cdots \circ g_{w_{-\left(j_{1}+1\right)}}^{-1}\left(U\left(z_{t}\right)\right) \cap U\left(z_{t}\right) \neq \varnothing$, which is a contradiction. Therefore, there exists $1 \leq j \leq m_{0}$ such that $G_{w}^{-j}(y) \notin K\left(\right.$ as $\left.G_{w}^{0}(y)=y \in K\right)$. From Case 1, it follows that there is an $\varepsilon$-chain $\left\{x_{0}=x, x_{1}, \ldots, x_{n}=G_{w}^{-j}(y)\right\}$ from $x$ to $G_{w}^{-j}(y)$. This implies that $\left\{x_{0}, x_{1}, \ldots, x_{n}=G_{w}^{-j}(y), G_{w}^{-(j-1)}(y), \ldots, G_{w}^{-1}(y), y\right\}$ is an $\varepsilon$-chain from $x$ to $y$, i.e., $y \in C_{\varepsilon}(x, G)$.

Therefore, $y \in C(x, G)$ as $\varepsilon$ is arbitrary.

Corollary 2.8. If the finitely generated semigroup $G$ is chain transitive on $\Omega(G)$, then it is chain transitive.

Proof. It follows directly from Proposition 2.7.

The following example shows that the assumption that the generators $g_{i}, i=1, \ldots, m$ are homemorphisms in Proposition 2.7 is necessary.

Example 2.9. Let $X=\{a, b, c\}$ with the discrete metric. Define two continuous maps $g_{1}, g_{2}: X \rightarrow X$ by $g_{1} \equiv a$ and $g_{2} \equiv b$, respectively. Let $G$ be the semigroup generated by $G_{1}=\left\{i d, g_{1}, g_{2}\right\}$. Then it is easy to see that $\Omega(G)=\{a, b\}$ and $a, b \in C(c, G)$. But $c \notin C(c, G)$, and thus $C(c, G) \neq X$.

Definition $2.10([16])$. A metric space $(X, d)$ is said to be generalized homogeneous if, for any $\varepsilon>0$, there exists $\delta>0$ such that if $\left\{\left(x_{1}, y_{1}\right), \ldots,\left(x_{n}, y_{n}\right)\right\}$ is a finite set of points in $X \times X$ satisfying:

(a) $\max _{1 \leq i \leq n} d\left(x_{i}, y_{i}\right)<\delta$,

(b) $x_{i} \neq x_{j}$ and $y_{i} \neq y_{j}$ for $1 \leq i \neq j \leq n$,

then there exists a homeomorphism $h: X \rightarrow X$ with $d_{0}(h, i d)<\varepsilon$ and $h\left(x_{i}\right)=y_{i}$ for $1 \leq i \leq n$. Here, we will call such $\delta$ an $\varepsilon$-modulus of homogeneity of $X$.

From [16], a topological manifold without boundary $(\operatorname{dim}(X) \geq 2)$, a Cartesian product of a countably infinite number of manifolds with nonempty boundary, and the Cantor set are all generalized homogeneous. 
By Remark 2.4, $\Omega_{\mathrm{w}}(G) \neq C R(G)$. In what follows, we shall show that $\Omega_{\mathrm{w}}(G)=C R(G)$ for a finitely generated semigroup $G$ on a generalized homogeneous space.

Proposition 2.11. Let $G$ be a semigroup generated by the finite family $\left\{i d, g_{1}, \ldots, g_{m}\right\}$ of continuous self-maps on a generalized homogeneous space $X$. Then

$$
\operatorname{Per}_{\mathrm{w}}(G)=\Omega_{\mathrm{w}}(G)=C R(G) .
$$

Proof. Fix $x \in C R(G)$. For any $\varepsilon>0$, choose $\delta>0$ as an $\varepsilon$-modulus of homogeneity of $X$. Take a $(\delta, w)$-chain $\Gamma=\left\{x_{i}\right\}_{i=0}^{k}$ from $x$ to $x$ for some $w=w_{0} \cdots w_{k-1} \in \mathcal{A}_{m}$. Without loss of generality, we may assume that $x_{i} \neq x_{j}$ and $g_{w_{i}}\left(x_{i}\right) \neq g_{w_{j}}\left(x_{j}\right)$ for $0 \leq i \neq j \leq k-1$. In fact, if there exists $0 \leq i<j \leq k-1$ such that $x_{i}=x_{j}$ or $g_{w_{i}}\left(x_{i}\right)=g_{w_{j}}\left(x_{j}\right)$, we can replace $\Gamma$ by $\left\{x_{0}, \ldots, x_{i}, x_{j+1}, \ldots, x_{k}\right\}$. This implies that the finite set

$$
\left\{\left(g_{w_{0}}(x), x_{1}\right),\left(g_{w_{1}}\left(x_{1}\right), x_{2}\right), \ldots,\left(g_{w_{k-1}}\left(x_{k-1}\right), x\right)\right\}
$$

satisfies the conditions (a) and (b) in Definition 2.10. Then, there exists a continuous map $h: X \rightarrow X$ with $d_{0}(h, i d)<\varepsilon$ such that $h\left(g_{w_{i}}\left(x_{i}\right)\right)=x_{i+1}$ for $0 \leq i \leq k-1$. Take $f_{i}=h \circ g_{i}(1 \leq i \leq m)$ and let $F$ be the semigroup generated by $\left\{i d, f_{1}, \ldots, f_{m}\right\}$. It can be verified that $F_{w}(x)=x$ and $D_{0}(G, F)<\varepsilon$, and hence $x \in \operatorname{Per}_{\mathrm{w}}(G)$. This, together with (2.2), implies that $\operatorname{Per}_{\mathrm{w}}(G)=\Omega_{\mathrm{w}}(G)=C R(G)$.

\section{Various type of recurrent sets on non-compact metric spaces}

The aim of this section is to present new notions for recurrent sets introduced in Definition 2.1 on the non-compact metric spaces, which are dependent of metrics.

Given a semigroup $G \in C_{m}(X)$ generated by $G_{1}=\left\{i d, g_{1}, \ldots, g_{m}\right\}$. Note that the definition of non-wandering point of the semigroup $G$ as in Definition 2.1 is independent of the choices of metrics for the space $X$. As we can see in the following, the definitions of weakly periodic points, weakly non-wandering points, and the set of chain recurrent points depend on the choices of metrics for non-compact space $X$.

Example 3.1. Define $T: \mathbb{R} \rightarrow \mathbb{S}^{1}$ by

$$
T(t)=\left(\frac{2 t}{1+t^{2}}, \frac{t^{2}-1}{t^{2}+1}\right) \text { for } t \in \mathbb{R},
$$

and let $X=T(\mathbb{Z})$. Also let $d^{\prime}$ be the metric on $X$ induced by the Riemannian metric on $\mathbb{S}^{1}$ and let $d$ be a discrete metric on $X$. It is clear that $d$ and $d^{\prime}$ induce the same topology on $X$. Define respectively $g_{1}, g_{2}: X \rightarrow X$ by $g_{1}\left(a_{i}\right)=a_{i+2}$ and $g_{2}\left(a_{i}\right)=a_{i+1}$, where $a_{i}=T(i)(i \in \mathbb{Z})$. Denote by $G$ the semigroup generated by $G_{1}=\left\{i d, g_{1}, g_{2}\right\}$. It is easy to see that $T(0) \in C R(G)$ with respect to the metric $d^{\prime}$, while $T(0) \notin C R(G)$ with respect to the metric $d$.

Now, fix $\varepsilon>0$ and choose $k \in \mathbb{N}$ satisfying $d^{\prime}\left(a_{k}, a_{-k}\right)<\varepsilon$. Consider a homeomorphism $f_{2}: X \rightarrow X$ which is defined by

$$
f_{2}\left(a_{i}\right)= \begin{cases}a_{i+1}, & i \in\{-k, \ldots, k-1\}, \\ a_{-k}, & i=k, \\ a_{i}, & \text { otherwise. }\end{cases}
$$

Take $f_{1}:=g_{1}$ and let $F$ be the semigroup generated by $\left\{i d, f_{0}, f_{1}\right\}$. By construction,

$$
D_{0}(F, G)=\max _{1 \leq i \leq 2} \max _{x \in X} d^{\prime}\left(f_{i}(x), g_{i}(x)\right)<\varepsilon .
$$

It is easy to see that $a_{0}=T(0) \in \operatorname{Per}(F)$, and thus $a_{0} \in P e r_{\mathrm{w}}(G) \subset \Omega_{\mathrm{w}}(G)$ with respect to the metric $d^{\prime}$, but $a_{0} \notin \Omega_{\mathrm{w}}(G)$ with respect to the metric $d$. Clearly, $a_{0} \notin \operatorname{Per}_{\mathrm{w}}(G)$ with respect to the metric $d$ by applying $\operatorname{Per}_{\mathrm{w}}(G) \subset \Omega_{\mathrm{w}}(G)$. 
Definition 3.2. Let $X$ and $Y$ be two metric spaces. We say that two semigroups $F$ and $G$ with generating sets $F_{1}=\left\{i d, f_{1}, \ldots, f_{m}\right\}$ and $G_{1}=\left\{i d, g_{1}, \ldots, g_{m}\right\}$ on $X$ and $Y$, respectively, are (topologically) conjugate if there exists a homeomorphism $h: X \rightarrow Y$ such that $h \circ f_{i}=g_{i} \circ h$ for all $1 \leq i \leq m$. The homeomorphism $h$ is called a conjugacy between $F$ and $G$.

For a semigroup $G \in C_{m}(X)$ and $\Lambda(G) \in\left\{\operatorname{Per}(G), \operatorname{Per}_{\mathrm{w}}(G), \Omega(G), \Omega_{\mathrm{w}}(G), C R(G)\right\}$, we say that $\Lambda(G)$ is preserved under conjugacy if, for every semigroup $F \in C_{m}(Y)$, which is conjugate to $G$ under some conjugacy $h: X \rightarrow Y$, we have $h(\Lambda(G))=\Lambda(F)$.

By Example 3.1, the definitions of $C R(G), \operatorname{Per}_{\mathrm{w}}(G)$, and $\Omega_{\mathrm{w}}(G)$ depend on the choices of metrics, and thus they are not preserved under conjugacy. In what follows, we introduce new notions of chain recurrent point, weakly periodic point, weakly non-wandering point for the semigroups on non-compact metric spaces, which are preserved under conjugacy. Also, they are all equivalent to the classical definitions in the case of compact metric spaces.

Let $\mathrm{C}(X)$ be the collection of all continuous functions from $X$ to $(0, \infty)$. For $\alpha, \beta \in \mathfrak{C}(X)$, we use the notation $\alpha<\beta$, whenever $\alpha(x)<\beta(x)$ for all $x \in X$.

Definition 3.3. Let $(X, d)$ be a metric space and $G, F \in C_{m}(X)$ be two semigroups generated by the finite families $G_{1}=\left\{i d, g_{1}, \ldots, g_{m}\right\}$ and $F_{1}=\left\{i d, f_{1}, \ldots, f_{m}\right\}$, respectively.

(i) For $w \in \mathcal{A}_{m}, x, y \in X$, and $\varepsilon \in \mathcal{C}(X)$, an $(\varepsilon, w)$-chain of semigroup $G$ from $x$ to $y$ is a finite sequence $\left\{x_{0}=x, x_{1}, \ldots, x_{n}=y\right\}$ such that $d\left(f_{w_{i}}\left(x_{i}\right), x_{i+1}\right)<\varepsilon\left(f_{w_{i}}\left(x_{i}\right)\right)$, for all $1 \leq i \leq n-1$.

(ii) For $\varepsilon \in \mathcal{C}(X)$, the semigroup $F$ is $\varepsilon$-close to $G$, denoted by $\mathcal{D}_{0}(F, G)<\varepsilon$, if $d\left(g_{i}(x), f_{i}(x)\right)<\min \left\{\varepsilon\left(g_{i}(x)\right), \varepsilon\left(f_{i}(x)\right)\right\}$ for all $1 \leq i \leq m$ and $x \in X$.

(iii) $x \in X$ is a chain recurrent point of semigroup $G$ if, for any $\varepsilon \in \mathcal{C}(X)$, there exists an $(\varepsilon, w)$-chain of the semigroup $G$ from $x$ to itself for some $w \in \mathcal{A}_{m}$.

(iv) $x \in X$ is weakly periodic point of the semigroup $G$ if, for any $\varepsilon \in \mathfrak{C}(X)$, there exists a semigroup $F^{\prime} \in C_{m}(x)$ such that $\mathcal{D}_{0}\left(G, F^{\prime}\right)<\varepsilon$ and $x \in \operatorname{Per}\left(F^{\prime}\right)$.

(v) $x \in X$ is weakly non-wandering point of the semigroup $G$ if, for any $\varepsilon \in \mathcal{C}(X)$, there exists a semigroup $F^{\prime} \in C_{m}(X)$ such that $\mathcal{D}_{0}\left(G, F^{\prime}\right)<\varepsilon$ and $x \in \Omega\left(F^{\prime}\right)$.

In what follows, the sets of chain recurrent points, weakly periodic points, and weakly non-wandering points of the semigroup $G$ in Definition 3.3 are also denoted by $C R(G)$, $\operatorname{Per}_{\mathrm{w}}(G)$, and $\Omega_{\mathrm{w}}(G)$, respectively.

Lemma 3.4 ([13, Lemmas 2.7 and 2.8]). Let $(X, d)$ and $\left(Y, d^{\prime}\right)$ be two metric spaces.

(i) A function $f$ from $X$ to $Y$ is continuous if and only if, for any $\varepsilon \in \mathcal{C}(Y)$, there exists $\delta \in \mathcal{C}(X)$ such that if $d(x, y)<\delta(x)(x, y \in X)$, then $d^{\prime}(f(x), f(y))<\varepsilon(f(x))$.

(ii) For any $\alpha \in \mathfrak{C}(X)$, there exists $\gamma \in \mathfrak{C}(X)$ such that

$$
\gamma(x) \leq \inf \{\alpha(z): z \in B(x, \gamma(x))\} .
$$

Remark 3.5. Suppose inequality (3.1) holds. It can be verified that

(1) $d(x, y)<\gamma(x)$ implies that $d(x, y)<\inf \{\alpha(z): z \in B(x, \gamma(x))\} \leq \min \{\alpha(x), \alpha(y)\}$;

(2) $d(x, y)<\gamma(y)$ implies that

$$
d(x, y)=d(y, x)<\inf \{\alpha(z): z \in B(y, \gamma(y))\} \leq \min \{\alpha(x), \alpha(y)\} .
$$

Thus, $d(x, y)<\max \{\gamma(x), \gamma(y)\}$ implies that $d(x, y)<\min \{\alpha(x), \alpha(y)\}$.

Lemma 3.6. Let $(X, d)$ be a metric space and let $f_{1}, \ldots, f_{m}: X \rightarrow X$ be continuous maps. Then, for every $\varepsilon \in \mathcal{C}(X)$, there exists $\delta \in \mathcal{C}(X)$ such that if $d(x, y)<\delta(x)$, then $d\left(f_{i}(x), f_{i}(y)\right)<\varepsilon\left(f_{i}(x)\right)$ for all $1 \leq i \leq m$.

Proof. Since each $f_{i}$ is continuous, from Lemma 3.4, it follows that for $\varepsilon \in \mathcal{C}(X)$, there exists $\delta_{i} \in \mathcal{C}(X)$ such that $d(x, y)<\delta_{i}(x)$ implies that $d\left(f_{i}(x), f_{i}(y)\right)<\varepsilon\left(f_{i}(x)\right)$. Take 
$\delta: X \rightarrow(0, \infty)$ by

$$
\delta(x)=\min \left\{\delta_{i}(x): 1 \leq i \leq m\right\} \text { for } x \in X .
$$

It can be verified that $\delta \in \mathcal{C}(X)$ satisfies the condition of the lemma.

Proposition 3.7. Let $F$ and $G$ be two semigroups generated by the finite family $F_{1}=$ $\left\{i d, f_{1}, \ldots, f_{m}\right\}$ and $G_{1}=\left\{i d, g_{1}, \ldots, g_{m}\right\}$ of continuous self-maps on the metric spaces $(X, d)$ and $(Y, \rho)$, respectively. If $F$ and $G$ are topologically conjugate with the conjugacy $h$ : $X \rightarrow Y$, then $h(\Lambda(F))=\Lambda(G)$, where $\Lambda:=C R, \Omega_{\mathrm{w}}, P e r_{\mathrm{w}}$ are defined in Definition 3.3.

Proof. Since $h$ is a homeomorphism, it suffices to check that $h^{-1}(\Lambda(G)) \subseteq \Lambda(F)$.

(1) Let $x \in C R(G)$ and $\varepsilon \in \mathcal{C}(X)$. Since $h^{-1}$ is continuous, by Lemma 3.4, there exists $\delta \in$ $\mathcal{C}(Y)$ such that that if $\rho(x, y)<\delta(x)(x, y \in Y)$, then $d\left(h^{-1}(x), h^{-1}(y)\right)<\varepsilon\left(h^{-1}(x)\right)$. From $x \in C R(G)$, it follows that there exists a $(\delta, w)$-chain $\left\{x_{0}=x, x_{1}, \ldots, x_{n}=x\right\}$ of $G$ from $x$ to itself for some $w \in \mathcal{A}_{m}$, implying that, for any $0 \leq i \leq n-1$,

$$
d\left(h^{-1} \circ g_{w_{i}}\left(x_{i}\right), h^{-1}\left(x_{i+1}\right)\right)<\varepsilon\left(h^{-1} \circ g_{w_{i}}\left(x_{i}\right)\right) .
$$

This, together with $h^{-1} \circ g_{w_{i}}=f_{w_{i}} \circ h^{-1}$, implies that $d\left(f_{w_{i}} \circ h^{-1}\left(x_{i}\right), h^{-1}\left(x_{i+1}\right)\right)<$ $\varepsilon\left(f_{w_{i}} \circ h^{-1}\left(x_{i}\right)\right)$. Thus, $\left\{h^{-1}(x), h^{-1}\left(x_{1}\right), \ldots, h^{-1}(x)\right\}$ is an $(\varepsilon, w)$-chain of $F$ from $h^{-1}(x)$ to itself, and therefore $h^{-1}(x) \in C R(F)$.

(2) Given any fixed $\varepsilon^{\prime} \in \mathcal{C}(X)$, by the continuity of $h^{-1}$ and Lemma 3.4, there exists $\varepsilon \in \mathcal{C}(Y)$ such that

$$
\text { if } \rho(x, y)<\varepsilon(x)(x, y \in Y) \text {, then } d\left(h^{-1}(x), h^{-1}(y)\right)<\varepsilon^{\prime}\left(h^{-1}(x)\right) .
$$

Fix $p \in \Omega_{\mathrm{w}}(G)$. From Definition 3.3, it follows that there exists a semigroup $G^{\prime} \in$ $C_{m}(Y)$ with the generators $\left\{i d, g_{1}^{\prime}, \ldots, g_{m}^{\prime}\right\}$ such that $\mathcal{D}_{0}\left(G, G^{\prime}\right)<\varepsilon$ and $p \in \Omega\left(G^{\prime}\right)$. Put $h^{-1} \circ g_{i}^{\prime} \circ h=f_{i}^{\prime}$. For $1 \leq i \leq m$, since $G$ is $\varepsilon$-close to $G^{\prime}$, we have $\rho\left(g_{i}(y), g_{i}^{\prime}(y)\right)<$ $\varepsilon\left(g_{i}(y)\right)$ for all $y \in Y$. This, together with (3.2), implies that

$$
\begin{aligned}
d\left(f_{i} \circ h^{-1}(y), f_{i}^{\prime} \circ h^{-1}(y)\right) & =d\left(h^{-1} \circ g_{i}(y), h^{-1} \circ g_{i}^{\prime}(y)\right) \\
& <\varepsilon^{\prime}\left(h^{-1} \circ g_{i}(y)\right)=\varepsilon^{\prime}\left(f_{i} \circ h^{-1}(y)\right) .
\end{aligned}
$$

This implies that the semigroup $F^{\prime}$ generated by $\left\{i d, f_{1}^{\prime}, \ldots, f_{m}^{\prime}\right\}$ is $\varepsilon^{\prime}$-close to $F$, as $h^{-1}(Y)=X$. Now, we show that $h^{-1}(p) \in \Omega\left(F^{\prime}\right)$. For any open neighborhood $U$ of $h^{-1}(p)$, it is clear that $h(U)$ is an open neighborhood of $p$, as $h$ is a homeomorphism. From $p \in \Omega\left(G^{\prime}\right)$, it follows that there exist $n \in \mathbb{N}$ and $\omega \in \Sigma_{m}$ such that $G_{\omega}^{\prime n}(h(U)) \cap$ $h(U) \neq \varnothing$. This, together with $G_{\omega}^{\prime n} \circ h=h \circ F_{\omega}^{\prime n}$, implies that

$$
\varnothing \neq G_{\omega}^{\prime n}(h(U)) \cap h(U)=h\left(F_{\omega}^{\prime n}(U)\right) \cap h(U)=h\left(F_{\omega}^{\prime n}(U) \cap U\right) .
$$

This means that $h^{-1}(p) \in \Omega\left(F^{\prime}\right)$. Thus, $h^{-1}(p) \in \Omega_{\mathrm{w}}(F)$, as $\varepsilon^{\prime}$ is arbitrary. That is $h^{-1}\left(\Omega_{\mathrm{w}}(G)\right) \subset \Omega_{\mathrm{w}}(F)$.

(3) Similar to the proof of (2), it can be verified that $h^{-1}\left(\operatorname{Per}_{\mathrm{w}}(G)\right) \subset \operatorname{Per}_{\mathrm{w}}(F)$.

In the following, we show that the set $C R(G)$ with new notion of Definition 3.3 is also closed.

Proposition 3.8. Let $G$ be a semigroup generated by the finite family $\left\{i d, g_{1}, \ldots, g_{m}\right\}$ of continuous self-maps on a metric space $(X, d)$. Then $C R(G)$ is a closed set.

Proof. Let $\left\{x_{n}\right\}_{n \in \mathbb{N}} \subseteq C R(G)$ and let $x_{n} \rightarrow y$ for some $y \in X$. For any $\delta \in \mathcal{C}(X)$, since $\delta: X \rightarrow(0, \infty)$ is continuous, there exists $\varepsilon_{0}>0$ such that

$$
\delta\left(B\left(y, \varepsilon_{0}\right)\right) \subseteq\left(\frac{\delta(y)}{2}, \frac{3 \delta(y)}{2}\right),
$$

and

$$
\delta\left(B\left(g_{i}(y), \varepsilon_{0}\right)\right) \subseteq\left(\frac{\delta\left(g_{i}(y)\right)}{2}, \frac{3 \delta\left(g_{i}(y)\right)}{2}\right) \text { for } 1 \leq i \leq m .
$$


Take $M \in \mathbb{N}$ such that $\varepsilon_{0}-\frac{\delta(y)}{2^{M+1}}>0$. By Lemma 3.4 , there exists $\gamma \in \mathcal{C}(X)$ such that

$$
\gamma(x) \leq \inf \left\{\frac{\delta(z)}{2^{M+2}}: z \in B(x, \gamma(x))\right\} \text { for all } x \in X .
$$

For $\beta \in \mathfrak{C}(X)$ with $\beta<\min \left\{\varepsilon_{0}, \gamma\right\}$, by applying Lemma 3.6, there exists $\alpha \in \mathfrak{C}(X)$ such that $d\left(x_{1}, x_{2}\right)<\alpha\left(x_{1}\right)$ implies that $d\left(g_{i}\left(x_{1}\right), g_{i}\left(x_{2}\right)\right)<\beta\left(g_{i}\left(x_{1}\right)\right)$ for $1 \leq i \leq m$ and $x_{1}, x_{2} \in X$. From $x_{n} \rightarrow y$, it follows that there exists $N_{1} \in \mathbb{N}$ such that, for any $n \geq N_{1}$,

$$
d\left(y, x_{n}\right)<\min \left\{\varepsilon_{0}-\frac{\delta(y)}{2^{M+1}}, \frac{\delta(y)}{2}, \alpha(y)\right\} .
$$

Applying $\frac{\delta\left(x_{n}\right)}{2^{M+2}} \rightarrow \frac{\delta(y)}{2^{M+2}}>0$ yields that there exists $N>N_{1}$ such that

$$
\text { for any } n \geq N, \frac{\delta\left(x_{n}\right)}{2^{M+2}}<\frac{\delta(y)}{2^{M+1}} \text {. }
$$

Since $x_{N} \in C R(G)$, there exists a $(\gamma, w)$-chain $\left\{y_{0}=x_{N}, y_{1}, \ldots, y_{\ell+1}=x_{N}\right\} \subset X$ from $x_{N}$ to itself for some $w \in \mathcal{A}_{m}$, i.e., for any $0 \leq i \leq \ell$,

$$
d\left(g_{w_{i}}\left(y_{i}\right), y_{i+1}\right)<\gamma\left(g_{w_{i}}\left(y_{i}\right)\right) .
$$

Since $x_{N} \in B\left(g_{w_{\ell}}\left(y_{\ell}\right), \gamma\left(g_{w_{\ell}}\left(y_{\ell}\right)\right)\right)$, applying (3.6) yields

$$
\gamma\left(g_{w_{\ell}}\left(y_{\ell}\right)\right) \leq \frac{\delta\left(x_{N}\right)}{2^{M+2}}
$$

which, together with (3.8) and (3.9), implies that

$$
d\left(g_{w_{\ell}}\left(y_{\ell}\right), x_{N}\right)<\gamma\left(g_{w_{\ell}}\left(y_{\ell}\right)\right) \leq \frac{\delta\left(x_{N}\right)}{2^{M+2}}<\frac{\delta(y)}{2^{M+1}} .
$$

This, together with $d\left(x_{N}, y\right)<\varepsilon_{0}-\frac{\delta(y)}{2^{M+1}}$, implies that $d\left(g_{w_{\ell}}\left(y_{\ell}\right), y\right)<\varepsilon_{0}$, i.e., $g_{w_{\ell}}\left(y_{\ell}\right) \in$ $B\left(y, \varepsilon_{0}\right)$, and hence by $(3.4)$

$$
\frac{\delta(y)}{2}<\delta\left(g_{w_{\ell}}\left(y_{\ell}\right)\right)<\frac{3 \delta(y)}{2} .
$$

By applying (3.7) and (3.9), it follows

$$
d\left(g_{w_{\ell}}\left(y_{\ell}\right), y\right) \leq d\left(g_{w_{i}}\left(y_{\ell}\right), x_{N}\right)+d\left(x_{N}, y\right)<\gamma\left(g_{w_{\ell}}\left(y_{\ell}\right)\right)+\frac{\delta(y)}{2} .
$$

Meanwhile, by applying (3.6) and (3.12), it follows

$$
\gamma\left(g_{w_{\ell}}\left(y_{\ell}\right)\right)+\frac{\delta(y)}{2}<\frac{\delta\left(g_{w_{\ell}}\left(y_{\ell}\right)\right)}{2^{M+2}}+\delta\left(g_{w_{\ell}}\left(y_{\ell}\right)\right) .
$$

This, together with (3.13), implies that

$$
d\left(g_{w_{\ell}}\left(y_{\ell}\right), y\right)<\frac{\delta\left(g_{w_{\ell}}\left(y_{\ell}\right)\right)}{2^{M+2}}+\delta\left(g_{w_{\ell}}\left(y_{\ell}\right)\right) \leq 2 \delta\left(g_{w_{\ell}}\left(y_{\ell}\right)\right) .
$$

On the other hand, $d\left(y, x_{N}\right)<\alpha(y)$ implies that

$$
d\left(g_{w_{0}}(y), g_{w_{0}}\left(x_{N}\right)\right)<\beta\left(g_{w_{0}}(y)\right)<\varepsilon_{0} .
$$

Then, $g_{w_{0}}\left(x_{N}\right) \in B\left(g_{w_{0}}(y), \varepsilon_{0}\right)$, and thus $\delta\left(g_{w_{0}}\left(x_{N}\right)\right)<\frac{3 \delta\left(g_{w_{0}}(y)\right)}{2}$ by applying (3.5). Therefore,

$$
\begin{aligned}
d\left(g_{w_{0}}(y), y_{1}\right) & \leq d\left(g_{w_{0}}(y), g_{w_{0}}\left(x_{N}\right)\right)+d\left(g_{w_{0}}\left(x_{N}\right), y_{1}\right) \\
& \leq \beta\left(g_{w_{0}}(y)\right)+\gamma\left(g_{w_{0}}\left(x_{N}\right)\right) \quad\left(\text { as } y_{0}=x_{N}\right) \\
& \leq \frac{\delta\left(g_{w_{0}}(y)\right)}{2^{M+2}}+\frac{\delta\left(g_{w_{0}}\left(x_{N}\right)\right)}{2^{M+2}} \quad\left(\text { as } \beta<\gamma \leq \frac{\delta}{2^{M+2}}\right) \\
& \leq \frac{\delta\left(g_{w_{0}}(y)\right)}{2^{M+2}}+\frac{3 \delta\left(g_{w_{0}}(y)\right)}{2^{M+3}}=\frac{5 \delta\left(g_{w_{0}}(y)\right)}{2^{M+3}}<\delta\left(g_{w_{0}}(y)\right) .
\end{aligned}
$$


This, together with (3.15), implies that the sequence $\left\{y_{0}=y, y_{1}, \ldots, y_{\ell}, y\right\}$ is a $(2 \delta, w)$ chain from $y$ to $y$. Hence $y \in C R(G)$, as $\delta \in \mathcal{C}(X)$ is arbitrary.

In the following, we study the relation between some notions in Definition 3.3. It is clear from the definition that $\operatorname{Per}(G) \subseteq \operatorname{Per}_{\mathrm{w}}(G) \subseteq \Omega_{\mathrm{w}}(G)$ and $\Omega(G) \subseteq \Omega_{\mathrm{w}}(G)$.

Proposition 3.9. Let $G \in C_{m}(X)$ be a semigroup generated by the finite family $\left\{i d, g_{1}, \ldots, g_{m}\right\}$ of continuous self-maps on a metric space $(X, d)$. Then $\Omega_{\mathrm{w}}(G) \subseteq C R(G)$.

Proof. Fix $p \in \Omega_{\mathrm{w}}(G)$. For any $\varepsilon \in \mathcal{C}(X)$, by Lemma 3.4, there exists $\varepsilon_{1} \in \mathcal{C}(X)$ such that $\varepsilon_{1}(x) \leq \inf \left\{\frac{\varepsilon(z)}{2}: z \in B\left(x, \varepsilon_{1}(x)\right)\right\}$. Let $\delta \in \mathcal{C}(X)$ such that $\delta(x) \leq \inf \left\{\varepsilon_{1}(z)\right.$ : $z \in B(x, \delta(x))\}$. From $p \in \Omega_{\mathrm{w}}(G)$, it follows that there exists a semigroup $F \in C_{m}(X)$ generated by $\left\{i d, f_{1}, \ldots, f_{m}\right\}$ such that $\mathcal{D}_{0}(G, F)<\delta$ and $p \in \Omega(F)$. Applying Lemma 3.6 yields that there exists $\delta_{1} \in \mathfrak{C}(X)$ with $\delta_{1}<\delta$ such that

if $d(x, y)<\delta_{1}(x)(x, y \in X)$, then $d\left(f_{i}(x), f_{i}(y)\right)<\delta\left(f_{i}(x)\right)$ for all $1 \leq i \leq m$.

This implies that there exist $n \in \mathbb{N}$ and $\omega \in \Sigma_{m}$ such that

$$
F_{\omega}^{n}\left(B\left(p, \delta_{1}(p)\right)\right) \cap B\left(p, \delta_{1}(p)\right) \neq \varnothing .
$$

Take $y \in B\left(p, \delta_{1}(p)\right)$ such that $F_{\omega}^{n}(y) \in B\left(p, \delta_{1}(p)\right)$.

We claim that $\left\{p, f_{\omega_{0}}(y), F_{\omega}^{2}(y), \ldots, F_{\omega}^{n-1}(y), p\right\}$ is an $(\varepsilon, w)$-chain of $G$ from $p$ to itself for some $w \in \mathcal{A}_{m}$. Since $d\left(g_{\omega_{0}}(p), f_{\omega_{0}}(p)\right)<\delta\left(g_{\omega_{0}}(p)\right)$ and $\delta(x) \leq \inf \left\{\varepsilon_{1}(z): z \in\right.$ $B(x, \delta(x))\}$, by Remark 3.5 , it follows that

$$
d\left(g_{\omega_{0}}(p), f_{\omega_{0}}(p)\right)<\min \left\{\varepsilon_{1}\left(g_{\omega_{0}}(p)\right), \varepsilon_{1}\left(f_{\omega_{0}}(p)\right)\right\} \leq \varepsilon_{1}\left(f_{\omega_{0}}(p)\right),
$$

i.e., $g_{\omega_{0}}(p) \in B\left(f_{\omega_{0}}(p)\right), \varepsilon_{1}\left(f_{\omega_{0}}(p)\right)$. This implies that

$$
\delta\left(f_{\omega_{0}}(p)\right) \leq \varepsilon_{1}\left(f_{\omega_{0}}(p)\right) \leq \frac{\varepsilon\left(g_{\omega_{0}}(p)\right)}{2} .
$$

Then,

$$
\begin{aligned}
d\left(g_{\omega_{0}}(p), f_{\omega_{0}}(y)\right) & \leq d\left(g_{\omega_{0}}(p), f_{\omega_{0}}(p)\right)+d\left(f_{\omega_{0}}(p), f_{\omega_{0}}(y)\right) \\
& <\delta\left(g_{\omega_{0}}(p)\right)+\delta\left(f_{\omega_{0}}(p)\right)\left(\text { by } d(p, y)<\delta_{1}(p) \text { and }(3.16)\right) \\
& <\frac{\varepsilon\left(g_{\omega_{0}}(p)\right)}{2}+\frac{\varepsilon\left(g_{\omega_{0}}(p)\right)}{2}=\varepsilon\left(g_{\omega_{0}}(p)\right) .
\end{aligned}
$$

From $F_{\omega}^{n}(y) \in B\left(p, \delta_{1}(p)\right) \subset B(p, \delta(p))$, it follows

$$
\delta(p)<\varepsilon_{1}\left(F_{\omega}^{n}(y)\right) .
$$

From $\mathcal{D}_{0}(F, G)<\delta$, it is easy to obtain that, for any $1 \leq i \leq n-1$,

$$
d\left(f_{\omega_{i}}\left(F_{\omega}^{i}(y)\right), g_{\omega_{i}}\left(F_{\omega}^{i}(y)\right)\right)<\delta\left(f_{\omega_{i}}\left(F_{\omega}^{i}(y)\right)\right) \leq \varepsilon_{1}\left(F_{\omega}^{i+1}(y)\right),
$$

and

$$
d\left(f_{\omega_{i}}\left(F_{\omega}^{i}(y)\right), g_{\omega_{i}}\left(F_{\omega}^{i}(y)\right)\right)<\delta\left(g_{\omega_{i}}\left(F_{\omega}^{i}(y)\right)\right) \leq \varepsilon\left(g_{\omega_{i}}\left(F_{\omega}^{i}(y)\right)\right) .
$$

In particular,

$$
d\left(f_{\omega_{n-1}}\left(F_{\omega}^{n-1}(y)\right), g_{\omega_{n-1}}\left(F_{\omega}^{n-1}(y)\right)\right) \leq \varepsilon_{1}\left(F_{\omega}^{n}(y)\right),
$$

i.e., $g_{\omega_{n-1}}\left(F_{\omega}^{n-1}(y)\right) \in B\left(f_{\omega_{n-1}}\left(F_{\omega}^{n-1}(y)\right), \varepsilon_{1}\left(F_{\omega}^{n}(y)\right)\right)$, by the choice of $\varepsilon_{1}$, it follows

$$
\varepsilon_{1}\left(F_{\omega}^{n}(y)\right)=\varepsilon_{1}\left(f_{\omega_{n-1}}\left(F_{\omega}^{n-1}(y)\right)\right) \leq \frac{\varepsilon\left(g_{\omega_{n-1}}\left(F_{\omega}^{n-1}(y)\right)\right)}{2} .
$$


This implies that

$$
\begin{aligned}
d\left(g_{\omega_{n-1}}\left(F_{\omega}^{n-1}(y)\right), p\right) & \leq d\left(g_{\omega_{n-1}}\left(F_{\omega}^{n-1}(y)\right), f_{\omega_{n-1}}\left(F_{\omega}^{n-1}(y)\right)\right)+d\left(F_{\omega}^{n}(y), p\right) \\
& \leq \delta\left(g_{\omega_{n-1}}\left(F_{\omega}^{n-1}(y)\right)+\delta_{1}(p) \quad\left(\text { as } \mathcal{D}_{0}(F, G)<\delta\right)\right. \\
& \leq \delta\left(g_{\omega_{n-1}}\left(F_{\omega}^{n-1}(y)\right)+\delta(p) \quad\left(\text { as } \delta_{1}<\delta\right)\right. \\
& <\frac{\varepsilon\left(g_{\omega_{n-1}}\left(F_{\omega}^{n-1}(y)\right)\right)}{2}+\varepsilon_{1}\left(F_{\omega}^{n}(y)\right) \quad(\text { by }(3.19)) \\
& =\varepsilon\left(g_{\omega_{n-1}}\left(F_{\omega}^{n-1}(y)\right)\right) \quad(\text { by }(3.22)) .
\end{aligned}
$$

Combining this with (3.18) and (3.21), it follows that $\left\{p, f_{\omega_{0}}(y), F_{\omega}^{2}(y), \ldots, F_{\omega}^{n-1}(y), p\right\}$ is an $\varepsilon$-chain of $G$ from $p$ to itself. Therefore, $p \in C R(G)$ as $\varepsilon$ is arbitrary.

\section{Shadowing and weak shadowing on compact metric spaces}

In this section, we introduce the notion of weak shadowing property for the finitely generated semigroup actions on the compact metric spaces and investigate the relationship between the shadowing and weak shadowing properties.

Let $(X, d)$ be a compact metric space and $H(X)$ be the collection of all homeomorphisms on $X$ with the following $C^{0}$-metric $d_{0}$ :

$$
d_{0}(f, g)=\max _{x \in X} d(f(x), g(x))+\max _{x \in X} d\left(f^{-1}(x), g^{-1}(x)\right) .
$$

Let $H_{m}(X)$ be the collection of all semigroups such as $G$ on the space $X$ which has a finite set of generators $\left\{i d, g_{1}, \ldots, g_{m}\right\}$, where $g_{i} \in H(X), i=1, \ldots, m$. Given two semigroups $F, G \in H_{m}(X)$ generated by the finite families $F_{1}=\left\{i d, f_{1}, \ldots, f_{m}\right\}$ and $G_{1}=\left\{i d, g_{1}, \ldots, g_{m}\right\}$, respectively. Defined the $C^{0}$-metric $D_{0}$ on $H_{m}(X)$ by

$$
D_{0}(F, G)=\max _{1 \leq i \leq m} d_{0}\left(f_{i}, g_{i}\right) .
$$

Definition 4.1. We say that a semigroup $G \in H_{m}(X)$ has the weak shadowing property if, for any $\varepsilon>0$, there exists $\delta(\varepsilon)>0$ such that, for any $F \in H_{m}(X)$ with $D_{0}(G, F)<\delta$, $x \in X$, and $\omega \in \Sigma_{m}$, there exists $y \in X$ such that

$$
d\left(G_{\omega}^{i}(y), F_{\omega}^{i}(x)\right)<\varepsilon \text { for all } i \in \mathbb{Z} .
$$

Clearly, the above definition of weak shadowing property for the semigroup action $G$ generated by $\left\{i d, g_{1}\right\}$ coincides with the notion of weak shadowing property for homeomorphisms in [4, Definition 4.2].

Definition 4.2. Let $G \in H_{m}(X)$ be a semigroup generated by $G_{1}=\left\{i d, g_{1}, \ldots, g_{m}\right\}$ on a compact metric space $(X, d)$ and $\delta>0$.

(1) The sequence $\left\{x_{i}\right\}_{i \in \mathbb{Z}}$ is a $(\delta, \omega)$-pseudo orbit of $G$ for some $\omega=\left(\ldots, \omega_{-1}, \omega_{0}, \omega_{1}, \ldots\right) \in$ $\Sigma_{m}$ if, for any $i \in \mathbb{Z}$,

$$
d\left(g_{\omega_{i}}\left(x_{i}\right), x_{i+1}\right)<\delta .
$$

(2) $G$ has the shadowing property if, for any $\varepsilon>0$, there exists $\delta>0$ such that every $(\delta, \omega)$-pseudo orbit $\left\{x_{i}\right\}_{i \in \mathbb{Z}}$ can be $\varepsilon$-shadowed by some point $z$ in $X$, that is,

$$
d\left(G_{\omega}^{i}(z), x_{i}\right)<\varepsilon \text { for all } i \in \mathbb{Z} .
$$

(3) $G$ has the finite shadowing property if, for any $\varepsilon>0$, there exists $\delta>0$ such that, for every $(\delta, w)$-chain $\left\{x_{i}\right\}_{i=0}^{n}$, there exists a point $z \in X$ such that

$$
d\left(G_{w}^{i}(z), x_{i}\right)<\varepsilon \text { for all } 0 \leq i \leq n .
$$

Remark 4.3. Note that, for a compact metric space $X$, we can define the shadowing and weak shadowing properties for the semigroup $G \in C_{m}(X)$, by replacing $\mathbb{Z}$ with $\mathbb{Z}^{+}$and using the $C_{0}$-metric on $C_{m}(X)$ defined in Section 2. 
It is well known that every mapping with the shadowing property has the weak shadowing property. In the following, we show that this is also true for the semigroup actions.

Lemma 4.4. Let $G \in H_{m}(X)$ be a semigroup generated by $G_{1}=\left\{i d, g_{1}, \ldots, g_{m}\right\}$ on a compact metric space $(X, d)$. If $G$ has the shadowing property, then it has the weak shadowing property.

Proof. Given any $\varepsilon>0$, choose $\delta>0$ as an $\varepsilon$-modulus of the shadowing property. For any $\omega \in \Sigma_{m}, x \in X$, and $F \in H_{m}(x)$ with $D_{0}(F, G)<\delta$, we have

$$
d\left(g_{\omega_{i}}\left(F_{\omega}^{i}(x)\right), F_{\omega}^{i+1}(x)\right)=d\left(g_{\omega_{i}}\left(F_{\omega}^{i}(x)\right), f_{\omega_{i}}\left(F_{\omega}^{i}(x)\right)<\delta \text { for all } i \in \mathbb{Z} .\right.
$$

This implies that $\left\{F_{\omega}^{i}(x)\right\}_{i \in \mathbb{Z}}$ is a $\delta$-pseudo orbit of $G$. Therefore, $\left\{F_{\omega}^{i}(x)\right\}_{i \in \mathbb{Z}}$ can be $\varepsilon$-shadowed by some point in $X$.

Lemma 4.5. Let $G \in H_{m}(X)$ be a semigroup generated by $G_{1}=\left\{i d, g_{1}, \ldots, g_{m}\right\}$ on a compact metric space $(X, d)$. If $G$ has the weak shadowing property, then each $g_{i}$ for $(1 \leq i \leq m)$ has the weak shadowing property.

Proof. For any $\varepsilon>0$, choose $\delta>0$ as an $\varepsilon$-modulus of the weak shadowing property. Fix $j \in\{1, \ldots, m\}$. Take $f_{i}=g_{i}$ for $i \in\{1, \ldots, m\} \backslash\{j\}$, and $f_{j} \in H(X)$ with $d_{0}\left(g_{j}, f_{j}\right)<\delta$. Let $F$ be the finitely generated semigroup with generators $\left\{i d, f_{1}, \ldots, f_{m}\right\}$. It can be verified that $D_{0}(G, F)<\delta$. Given $x \in X$, since $G$ has the weak shadowing property, for $\omega=(\ldots, j, j, j, \ldots) \in \Sigma_{m}$, there exists $y \in X$ such that

$$
d\left(G_{\omega}^{i}(y), F_{\omega}^{i}(x)\right)=d\left(g_{j}^{i}(y), f_{j}^{i}(x)\right)<\varepsilon \text { for all } i \in \mathbb{Z} .
$$

This means that $g_{j}$ has the weak shadowing property.

The following example is related to [4, Remark 4.4].

Example 4.6. In this example, we present a finitely generated semigroup with the weak shadowing property that does not have the shadowing property. Let $g:[0,2] \rightarrow[0,2]$ be defined by

$$
g(x)= \begin{cases}\sqrt{x}, & x \in[0,1], \\ \sqrt{x-1}+1, & x \in[1,2] .\end{cases}
$$

Fix $x_{0} \in(1,2)$ and define $g_{0}$ on $X=[0,1] \cup\left\{g^{n}\left(x_{0}\right): n \in \mathbb{Z}\right\} \cup\{2\}$ by $g_{0}=\left.g\right|_{X}$. Let $g_{1}: X \rightarrow X$ be defined by

$$
g_{1}(x)= \begin{cases}\sqrt[3]{x}, & x \in[0,1], \\ g_{0}(x), & x \in X \backslash[0,1]\end{cases}
$$

Then $g_{0}, g_{1}$ are homeomorphisms on the compact metric space $X$, with fixed points $0,1,2$. Denote $G$ the semigroup action with the finite set of generator $\left\{i d, g_{0}, g_{1}\right\}$. Then $G$ has the weak shadowing property. Indeed, let $F$ be a finitely generated semigroup associated with $\left\{i d, f_{0}, f_{1}\right\}$, which is sufficiently closed to $G$. We have to note that the homeomorphisms $f_{0}, f_{1}$ have to fix $0,1,2$. Take any point $x \in[0,1]$. Let $b_{1}<b_{2}$ be another fixed points of the mappings $f_{0}$ and $f_{1}$, respectively, which are closed to 1 (or 0 ). It is easy to see that the map $F_{\omega}^{i}$ has a fixed point $b \in\left[b_{1}, b_{2}\right]$ for any $\omega \in \Sigma_{m}$ and any $i \in \mathbb{Z}$. Indeed, the homeomorphisms $f_{0}$ and $f_{1}$ move any points of interval $\left[b_{2}, 1\right]$ to the right, while the accumulation points 1 is fixed. Therefore, we can choose $y \in[0,1]$ such that $G_{\omega}^{i}(y)$ is closed to $F_{\omega}^{i}(x)$. A similar argument is used for any $x \in[1,2]$. Thus, $G$ has the weak shadowing property, but it does not have the shadowing property, since $g_{0}$ and $g_{1}$ does not have the shadowing property. Indeed, there are pseudo-orbits starting at 0 and finishing at 2 without real orbits tracing them. 
By Lemma 4.4 and Example 4.6, the shadowing property is strictly stronger than the weak shadowing property finitely generated semigroups on compact metric spaces. Here, we shall show that the converse also holds for finitely generated semigroups on generalized homogeneous spaces.

Proposition 4.7. Let $(X, d)$ be a generalized homogeneous space without isolated points. Then $G \in H_{m}(X)$ has the shadowing property if and only if it has the weak shadowing property.

Proof. Necessity: By Lemma 4.4, this holds trivially.

Sufficiency: By [21, Lemma 3.3], it suffices to show that $G$ has the finite shadowing property. Without loss of generality, assume that $G$ is generated by $\left\{i d, g_{1}, \ldots, g_{m}\right\}$. For any fixed $\varepsilon>0$, choose $0<\delta<\frac{\varepsilon}{2}$ such that, for any $\hat{F} \in H_{m}(X)$ with $D_{0}(G, \hat{F})<\delta$, $x \in X$, and $\omega \in \Sigma_{m}$, there exists $y \in X$ such that $d\left(G_{\omega}^{i}(y), \hat{F}_{\omega}^{i}(x)\right)<\frac{\varepsilon}{2}$ for all $i \in \mathbb{Z}$. Take $\eta<\delta$ be a $\delta$-modulus of homogeneity of $X$. Since $g_{1}, \ldots, g_{m}$ are uniformly continuous, there exists $0<\hat{\eta}<\frac{\eta}{3}$ such that for any $x, y \in X$ with $d(x, y)<\hat{\eta}, d\left(g_{i}(x), g_{i}(y)\right)<\frac{\eta}{3}$ for all $1 \leq i \leq m$. Let $\left\{x_{i}\right\}_{0 \leq i<k}$ be an $(\hat{\eta}, w)$-pseudo orbit of $G$ with $w=w_{0} \cdots w_{k-1} \in \mathcal{A}_{m}$, that is, $d\left(g_{w_{i}}\left(x_{i}\right), x_{i+1}\right)<\hat{\eta}$ for $0 \leq i \leq k-1$. Since $X$ does not contain isolated points, we can choose $y_{0}=x_{0}, y_{1} \in B\left(x_{1}, \hat{\eta}\right) \backslash\left\{g_{w_{1}}^{-1} \circ g_{w_{0}}\left(y_{0}\right)\right\}$,

$$
\begin{gathered}
y_{2} \in B\left(x_{2}, \hat{\eta}\right) \backslash\left\{y_{1}, g_{w_{2}}^{-1} \circ g_{w_{1}}\left(y_{1}\right), g_{w_{2}}^{-1} \circ g_{w_{0}}\left(y_{0}\right)\right\}, \\
\vdots \\
y_{i} \in B\left(x_{i}, \hat{\eta}\right) \backslash\left\{y_{1}, \ldots, y_{i-1}, g_{w_{i}}^{-1} \circ g_{w_{i-1}}\left(y_{i-1}\right), \ldots, g_{w_{i}}^{-1} \circ g_{w_{0}}\left(y_{0}\right)\right\}, \\
\vdots \\
y_{k-1} \in B\left(x_{k-1}, \hat{\eta}\right) \backslash\left\{y_{1}, \ldots, y_{k-2}, g_{w_{k-1}}^{-1} \circ g_{w_{k-2}}\left(y_{k-2}\right), \ldots, g_{w_{k-1}}^{-1} \circ g_{w_{0}}\left(y_{0}\right)\right\}, \\
y_{k} \in B\left(x_{k}, \hat{\eta}\right) \backslash\left\{y_{1}, \ldots, y_{k-1}\right\} .
\end{gathered}
$$

It is easy to see that the set $\left\{\left(g_{w_{0}}\left(y_{0}\right), y_{1}\right),\left(g_{w_{1}}\left(y_{1}\right), y_{2}\right), \ldots,\left(g_{w_{k-1}}\left(y_{k-1}\right), y_{k}\right)\right\} \subset X \times X$ satisfies the followings:

a) $d\left(g_{w_{i}}\left(y_{i}\right), y_{i+1}\right) \leq d\left(g_{w_{i}}\left(y_{i}\right), g_{w_{i}}\left(x_{i}\right)\right)+d\left(g_{w_{i}}\left(x_{i}\right), x_{i+1}\right)+d\left(x_{i+1}, y_{i+1}\right)<\frac{\eta}{3}+\hat{\eta}+\hat{\eta}<\eta$ for $0 \leq i \leq k-1$;

b) $g_{w_{i}}\left(y_{i}\right) \neq g_{w_{j}}\left(y_{j}\right)$ and $y_{i+1} \neq y_{j+1}$ for $0 \leq i \neq j \leq k-1$.

Since $X$ is a generalized homogeneous space, there exists a homeomorphism $h: X \rightarrow X$ with $d_{0}(h, i d)<\delta$ and $h\left(g_{w_{i}}\left(y_{i}\right)\right)=y_{i+1}$ for $0 \leq i \leq k-1$. Take $f_{w_{i}}:=h \circ g_{w_{i}}$. It is easy to see that, for any $0 \leq i \leq k, f_{w}^{i}\left(y_{0}\right)=y_{i}$ and $d_{0}\left(g_{w_{i}}, f_{w_{i}}\right)<\delta$. For $\ell \in\{1, \ldots, m\} \backslash\left\{w_{0}, \ldots, w_{k-1}\right\}$, take $f_{\ell}=g_{\ell}$ and let $F$ be the semigroup generated by $\left\{i d, f_{1}, \ldots, f_{m}\right\}$. Clearly, $F$ is $\delta$-close to $G$. By the weak shadowing property of $G$, there exists a point $z \in X$ such that, for any $0 \leq i \leq k, d\left(G_{w}^{i}(z), F_{w}^{i}\left(y_{0}\right)\right)<\frac{\varepsilon}{2}$, implying that

$$
d\left(G_{w}^{i}(z), x_{i}\right) \leq d\left(G_{w}^{i}(z), F_{w}^{i}\left(y_{0}\right)\right)+d\left(F_{w}^{i}\left(y_{0}\right), x_{i}\right)<\frac{\varepsilon}{2}+d\left(y_{i}, x_{i}\right)<\frac{\varepsilon}{2}+\hat{\eta}<\varepsilon .
$$

Acknowledgment. This work was supported by the National Natural Science Foundation of China (No. 11601449), the Key Natural Science Foundation of Universities in Guangdong Province (No. 2019KZDXM027), the Science and Technology Innovation Team of Education Department of Sichuan for Dynamical System and its Applications (No. 18TD0013), the Youth Science and Technology Innovation Team of Southwest Petroleum University for Nonlinear Systems (No. 2017CXTD02), and the National Natural Science Foundation of China (Key Program) (No. 51534006). 


\section{References}

[1] S.A. Ahmadi, X. Wu, and G. Chen, Topological chain and shadowing properties of dynamical systems on uniform spaces, Topology Appl. 275, 107153, 2020.

[2] J. Ahn, K. Lee, and S. Lee, Persistent actions on compact metric spaces, J. Chungcheong Math. Soc. 30 (1), 61-66, 2017.

[3] D.V. Anosov, On a certain class of invariant sets of smooth dynamical systems, Proc. 5th Int. Conf. on Non-Linear Oscillations, 2, 39-45, 1970.

[4] A. Artigue, Lipschitz perturbations of expansive systems, Discrete Contin. Dyn. Syst. 35 (5), 1829-1841, 2015.

[5] A.Z. Bahabadi, Shadowing and average shadowing properties for iterated function systems, Georgian Math. J. 22 (2) 179-184, 2015.

[6] R. Bowen, Equilibrium states and ergodic theory of Anosov diffeomorphisms, Lecture Notes in Mathematics, 470, Springer-Verlag, Berlin-New York, 1975.

[7] M. Carvakho, F.B. Rodrigues, and P. Varandas, A variational principle for free semigroup actions, Adv. Math. 334, 450-487, 2018.

[8] S.K. Choi, C. Chu, and K. Lee, Recurrence in persistent dynamical systems, Bull. Aust. Math. Soc. 43 (3), 509-517, 1991.

[9] N. Chung and K. Lee, Topological stability and pseudo orbit tracing property of group actions, Proc. Amer. Math. Soc. 146 (3), 1047-1057, 2018.

[10] Z. Ding, J. Yin, and X. Luo, The multi-transitivity of free semigroup actions, Stoch. Dyn. 20 (5), 2050040, 2020.

[11] A. Fakhari and F.H. Ghane, On shadowing: ordinary and ergodic, J. Math. Anal. Appl. 364 (1), 151-155, 2010.

[12] Y. Ju, D. Ma and Y. Wang, Topological entropy of free semigroup actions for noncompact sets, Discrete Contin. Dyn. Syst. 39 (2), 995-1017, 2019.

[13] K. Lee, N. Nguyen, and Y. Yang, Topological stability and spectral decomposition for homeomorphisms on noncompact spaces, Discrete Contin. Dyn. Syst. 38 (5), 2487$2503,2018$.

[14] J. Lewowics, Persistence in expansive systems, Ergodic Theory Dynam. Systems, 3 (4), 567-578, 1983.

[15] Y. Liang and C. Zhao, Rates of recurrence for free semigroup actions, J. Dyn. Control Syst. 27, 417-425, 2021.

[16] M. Mazure, Weak shadowing for discrete dynamical systems on nonsmooth manifolds, J. Math. Anal. Appl. 281 (2), 657-662, 2003.

[17] P. Oprocha and X. Wu, On averaged tracing of periodic average pseudo orbits, Discrete Contin. Dyn. Syst. 37 (9), 4943-4957, 2017.

[18] A.V. Osipov and S.B. Tikhomirov, Shadowing for actions of some finitely generated groups, Dyn. Syst. 29 (3), 337-351, 2014.

[19] S.Y. Pilyugin, A.A. Rodionova, and K. Sakai, Orbital and weak shadowing properties, Discrete Contin. Dyn. Syst. 9 (2), 287-308, 2003.

[20] K. Sakai, Diffeomorphisms with the average-shadowing property on two-dimensional closed manifolds, Rocky Mountain J. Math. 30 (3), 1129-1137, 2000.

[21] Z. Shabani, Ergodic shadowing of semigroup actions, Bull. Iranian Math. Soc. 46 (2), 303-321, 2020.

[22] X. Wu, Chaos of transformations induced onto the space of probability measures, Internat. J. Bifur. Chaos 26 (13), 1650227, 2016.

[23] X. Wu, S. Liang, Y. Luo, M. Xin, and X. Zhang, A remark on the limit shadowing property for iterated function systems, Politehn. Univ. Bucharest Sci. Bull. Ser. A Appl. Math. Phys. 81 (3), 107-114, 2019.

[24] X. Wu, X. Ma, Z. Zhu, and T. Lu, Topological ergodic shadowing and chaos on uniform spaces, Internat. J. Bifur. Chaos 28 (3), 1850043, 2018. 
[25] X. Wu, P. Oprocha, and G. Chen, On various definitions of shadowing with average error in tracing, Nonlinearity, 29 (7), 1942-1972, 2016.

[26] X. Wu, L. Wang, and J. Liang, The chain properties and average shadowing property of iterated function systems, Qual. Theory Dyn. Syst. 17 (1), 219-227, 2018.

[27] X. Wu, X. Zhang, and X. Ma, Various shadowing in linear dynamical systems, Internat. J. Bifur. Chaos 29 (3), 1950042, 2019.

[28] X. Wu, X. Zhang, and F. Sun, Volume-preserving diffeomorphisms with the $\mathscr{M}_{0}$ shadowing properties, Mediterr. J. Math. 18, 45, 2021.

[29] X. Zhang and X. Wu, Diffeomorphisms with the $\mathscr{M}_{0}$-shadowing property, Acta Math. Sin. (Engl. Ser.) 35 (11), 1760-1770, 2019.

[30] X. Zhang, X. Wu, Y. Luo, and X. Ma, A remark on limit shadowing for hyperbolic iterated function systems, Politehn. Univ. Bucharest Sci. Bull. Ser. A Appl. Math. Phys. 81 (3), 139-146, 2019. 